\title{
Cuidados paliativos: conhecimento dos formandos de Medicina de uma instituição de ensino superior de Goiás
}

\author{
Palliative care: knowledge of graduates from a medical school in Goiás
}

\author{
Natália Sousa Costa' (1) natalia0611 costa@gmail.com \\ Nathália Maria Fonseca' (1) nathaliamfonseca@outlook.com \\ Isabella Augusto Santos' (D) isabella.augustomeed@gmail.com \\ Gustavo Marques Paulino' (D) gustavomarquesp11@gmail.com \\ Júlia Oliveira Carvalho' (1) juoliveiracarvalho06@gmail.com \\ Aila Davis Fanstone Pina Vieira' (D) ailadavis@hotmail.com
}

\section{RESUMO}

Introdução: Os cuidados paliativos (CP) consistem na assistência multidisciplinar e holística ao paciente para melhoria de sua qualidade de vida e de seus parentes, por meio da prevenção e do alívio de enfermidades, de modo a amenizar seus aspectos físicos, sociais, psicológicos e espirituais. Ressalta-se a crescente necessidade de CP, posto que, com o envelhecimento populacional e o aumento da prevalência de doenças crônicas não transmissíveis, há, no mundo, milhões de pessoas que necessitam de CP por ano, das quais apenas $14 \%$ recebem esses cuidados até o final da vida.

Objetivo: Este estudo teve como objetivo verificar o conhecimento dos formandos de Medicina de 2020 de uma instituição de ensino superior do interior de Goiás acerca dos CP.

Método: Este trabalho foi feito por meio de um estudo descritivo, transversal e de natureza quantitativa, no qual se utilizou o Questionário Geral sobre Cuidados Paliativos (QGCP), com adaptações e perguntas objetivas. Aplicou-se o QGCP de forma on-line. O universo amostral foi constituído por 74 formandos. As participações foram de caráter voluntário e, exclusivamente, para fins científicos, respaldando as recomendações éticas de anonimato e confidencialidade.

Resultado: Notou-se que a maioria dos participantes considerou os CP como "importantes" ou "muito importantes", embora uma minoria deles tenha se interessado em realizar alguma formação específica nessa área em sua prática clínica. Além disso, apesar de apenas uma questão do questionário aplicado ter apresentado mais de $50 \%$ de erro, inferindo que os participantes demonstram um certo conhecimento em $C P, 86,5 \%$ $(n=64)$ dos avaliados não consideraram seu conhecimento acerca de CP como "apropriado" ou "muito apropriado".

Conclusão: Os resultados revelam que, embora os acadêmicos reconheçam a importância dos $C P$, o desconhecimento acerca da sua filosofia e de seus princípios e a insegurança quanto às condutas a serem prescritas destacam a necessidade de uma educação permanente sobre o assunto. Esses achados corroboram estudos nacionais e internacionais que afirmam a necessidade da disseminação do conhecimento sobre a filosofia e os princípios dos CP.

Palavras-chave: Cuidados Paliativos; Faculdades de Medicina; Conhecimento.

\section{ABSTRACT}

Introduction: Palliative care (PC) consists of multidisciplinary and holistic assistance to the patient to improve their quality of life and that of their relatives, through the prevention and relief of illnesses, seeking to soften their physical, social, psychological and spiritual aspects. There is an outstanding and growing need for PC, given that, with an aging population and increased prevalence of chronic non-communicable diseases, millions of people require $P C$ every year, and only $14 \%$ of those people actually receive such services care until the end of life.

Objective: To gauge the knowledge of medical graduates in 2020 from a Higher Education Institution in the state of Goiás about PC.

Method: This work was conducted through a descriptive, transversal and quantitative study, using the validated questionnaire "General Questionnaire on Palliative Care - QGCP", containing adaptations and objective questions, performed online. The sample universe consisted of 74 graduates. Participation was voluntary and, exclusively for scientific purposes, in line with the ethical recommendations for anonymity and confidentiality.

Results: It was found that the majority of the participants considered PC to be "important" or "very important", although only a minority were interested in undergoing specific training in this area in their clinical practice. Furthermore, although only one question in the questionnaire was answered incorrectly more than $50 \%$ of the time, inferring that the participants demonstrate a certain knowledge in PC, 86.5\% ( $n=64$ ) of the participants did not consider their knowledge about PC as "appropriate" or "very appropriate".

Conclusion: The results reveal that although academics recognize the importance of PC, a lack of knowledge about the underlying philosophy and principles, as well as insecurity regarding the conducts to be prescribed, highlight the need for permanent education on the subject. These findings corroborate national and international studies, which affirm the need to disseminate knowledge about the philosophy and principles of PC.

Keywords: Palliative Care; Medical School; Knowledge.

${ }^{1}$ Universidade Evangélica de Goiás, Anápolis, Goiás, Brasil.

Editora-chefe: Rosiane Viana Zuza Diniz.

Editor associado: Antonio da Silva Menezes Junior.

Recebido em 16/07/21; Aceito em 01/09/21. | Avaliado pelo processo de double blind review. 


\section{INTRODUÇÃO}

Segundo a Organização Mundial da Saúde (OMS)',

Cuidados paliativos (CP) consistem na assistência promovida por uma equipe multidisciplinar, com o objetivo de melhorar a qualidade de vida do paciente e de seus familiares, diante de uma doença que ameace a vida. Isso deve ser feito por meio da prevenção e do alívio do sofrimento, da identificação precoce, da avaliação impecável e do tratamento da dor e dos demais sintomas físicos, sociais, psicológicos e espirituais.

De acordo com a OMS, 40 milhões de pessoas necessitam de cuidados paliativos (CP) por ano, mas apenas $14 \%$ recebem os cuidados até o final da vida. Além disso, a necessidade de CP torna-se cada vez mais frequente em razão do envelhecimento populacional e do aumento da prevalência de doenças crônicas não transmissíveis².

Segundo a Academia Nacional de Cuidados Paliativos (ANCP), mais da metade dos serviços de CP iniciaram as atividades na década de 2010, constatando o quanto a inserção dos CP no país é recente, de modo que ratifica um dos motivos das inúmeras falhas na sua aplicabilidade ${ }^{3}$.

Ainda, a falta de preparo para enfrentar a finitude da vida gera problemas na comunicação e no suporte aos pacientes, $\mathrm{o}$ que implica prejuízo na relação médico-paciente. Com o passar do tempo, o profissional se sente fracassado, pois não consegue dar o amparo necessário à pessoa, além da possibilidade de iatrogenia, em que o profissional prejudica o paciente com o excesso de cuidado4.

Levando em consideração essas dificuldades, éimportante iniciar o processo de ensino-aprendizagem acerca do tema ainda no período de graduação em Medicina ${ }^{2}$. Como exemplificação, um estudo realizado com estudantes de Medicina e Enfermagem abordou a dificuldade que eles enfrentam em relação ao tema, uma vez que as escolas médicas brasileiras (EMB) apresentam deficiência em conteúdos sobre o paliativismo 4 .

Tendo em vista a recente promoção da medicina paliativa como especialidade no Brasil e sabendo que as habilidades em CP são mais bem desempenhadas por discentes que adquirem treinamentos teóricos e práticos, é necessário conhecer os desafios envolvidos ${ }^{5}$. Além disso, sabe-se que a educação em paliativismo não ocorre continuadamente, já que a maior abordagem está inserida em disciplinas ligadas aos seguintes temas: morte, dor e tratamento oncológico. Assim, ainda carece de um direcionamento específico da especialidade por tratarse de um tema novo ${ }^{6}$.

Em razão da necessidade de haver médicos aptos a cuidar de pessoas com doenças crônicas ou terminais de maneira adequada, a presente pesquisa teve por objetivo verificar o conhecimento dos formandos de Medicina de 2020 de uma instituição de ensino superior do interior de Goiás acerca dos CP.

\section{MÉTODO}

\section{Tipo e local de estudo}

Trata-se de um estudo transversal, descritivo, de natureza quantitativa, desenvolvido com os formandos de Medicina de 2020 de uma instituição de ensino superior do interior de Goiás.

\section{População e amostra}

Com base no quadro geral de formandos de Medicina de 2020 da instituição pesquisada, o universo amostral foi constituído de 74 participantes. Deve-se destacar que se tratou de uma amostra por conveniência. Enviou-se o questionário a todos os formandos do ano de $2020(n=104)$, dos quais 74 responderam a ele de forma completa.

\section{Critérios de inclusão e exclusão}

Adotaram-se os seguintes critérios de inclusão: indivíduos de ambos os sexos deveriam ter concluído, em 2020, a graduação em Medicina na instituição analisada, aceitar por livre e espontânea vontade responder ao questionário e assinar o Termo de Consentimento Livre e Esclarecido (TCLE). Excluíram-se os questionários com informações incompletas.

\section{Coleta de dados}

Realizou-se a coleta de dados a partir de um questionário on-line enviado para os e-mails de todos os formandos de Medicina de 2020. Para a pesquisa, os e-mails foram fornecidos pela própria instituição, e efetuou-se a coleta no período de outubro de 2020 a fevereiro de 2021. Ao clicar no link enviado, o participante teve acesso à página do Google Forms que continha o TCLE e o título "Questionário Geral sobre Cuidados Paliativos - QGCP"7 referente ao instrumento modificado para a presente pesquisa. A primeira parte referese aos dados pessoais do participante e às dez questões de múltipla escolha que abordam o contato do médico com os CP. A segunda parte continha 30 perguntas objetivas, nas quais os participantes deveriam assinalar verdadeiro ou falso sobre o tema.

\section{Análise de dados}

Os dados foram transcritos para planilha em Programa MS Excel Office XP, e realizou-se estatística descritiva com os resultados expressos em frequências absoluta e relativa. 


\section{Aspectos éticos}

Oestudo foi conduzido conforme a Resolução n 466/2012 da Universidade Evangélica de Goiás (UniEvangélica) e aprovado pelo Comitê de Ética em Pesquisa: Parecer no 4.359.994.

\section{RESULTADOS}

Participaram deste estudo 74 médicos formados pela instituição pesquisada: $50 \%(n=37)$ do sexo feminino e $50 \%$ $(n=37)$ do sexo masculino, com idades variando entre 22 e 30 anos, com média de 25 anos.

Os dados referentes à formação em CP durante a graduação estão organizados na Tabela 1. Assim, 55,4\% $(n=41)$ dos alunos informaram que receberam formação sobre CP durante a graduação, entretanto apenas 14,9\% (n =11) realizaram trabalho na área. Dentre estes, alguns dos trabalhos realizados foram cuidados destinados a pacientes oncológicos e idosos, além de estágios em medicina de família e comunidade (MFC). Ademais, ainda que a maioria tenha recebido formação em $C P, 86,5 \%(n=64)$ não consideram a informação recebida suficiente.

Em relação aos participantes que receberam formação extracurricular sobre $\mathrm{CP}, 32,4 \%(\mathrm{n}=24)$ afirmaram ter participado de conferências, ação de formação, workshops, jornadas e cursos breves. Já $67,6 \%(n=50)$ negaram ter tido formação extracurricular na área dos $\mathrm{CP}$, sendo o principal motivo a pouca oferta formativa na área, além de ausência de conhecimento sobre formações na área, falta de tempo e falta de interesse.

A Tabela 2 retrata a visão do participante em relação à inclusão de $\mathrm{CP}$ na graduação médica. Dessa maneira, as tabelas 1 e 2, a partir das frequências absoluta e relativa, expressam que a maioria informou ter recebido formação sobre $C P$ na graduação, dos quais $71,6 \%(n=53)$ consideram muito importante a inclusão de conteúdos sobre $\mathrm{CP}$ no bacharelado de Medicina.

O Gráfico 1 representa a classificação do conhecimento que os formandos possuem em relação aos CP. Dessa forma, levando em conta aqueles que receberam formação sobre $\mathrm{CP}$ na graduação $(55,4 \%, n=41)$, a maioria classificou o próprio conhecimento como "razoável" $(65,8 \%, \mathrm{n}=27)$, ao passo que $19,5 \%(n=8)$ consideraram como "pouco apropriado". No que

Tabela 1. Formação em cuidados paliativos durante a faculdade.

\begin{tabular}{lcc}
\hline \multicolumn{1}{c}{ Itens avaliados } & $\mathbf{n}$ & $\%$ \\
\hline $\begin{array}{l}\text { No curso de licenciatura em Medicina, } \\
\text { recebeu formação sobre cuidados } \\
\text { paliativos. }\end{array}$ & 41 & 55,4 \\
$\begin{array}{l}\text { No curso de licenciatura em Medicina, } \\
\text { realizou algum trabalho na área dos } \\
\text { cuidados paliativos. }\end{array}$ & 11 & 14,9 \\
$\begin{array}{l}\text { Considera que, durante o curso, recebeu } \\
\text { informação suficiente sobre cuidados } \\
\text { destinados a doentes em situação } \\
\text { terminal. }\end{array}$ & 10 & 13,5 \\
$\begin{array}{l}\text { Frequentou formação extracurricular na } \\
\text { área de cuidados paliativos. }\end{array}$ & 24 & 32,4 \\
\hline
\end{tabular}

Tabela 2. Importância da inclusão de conteúdos de cuidados paliativos no curso de Medicina.

\begin{tabular}{ccc}
\hline Itens avaliados & $\mathbf{n}$ & $\%$ \\
\hline Nada importante & 0 & 0,0 \\
Pouco importante & 1 & 1,4 \\
Indiferente & 0 & 0,0 \\
Importante & 20 & 27,0 \\
Muito importante & 53 & 71,6 \\
\hline
\end{tabular}

Gráfico 1.Como classifica o conhecimento que possui sobre cuidados paliativos?

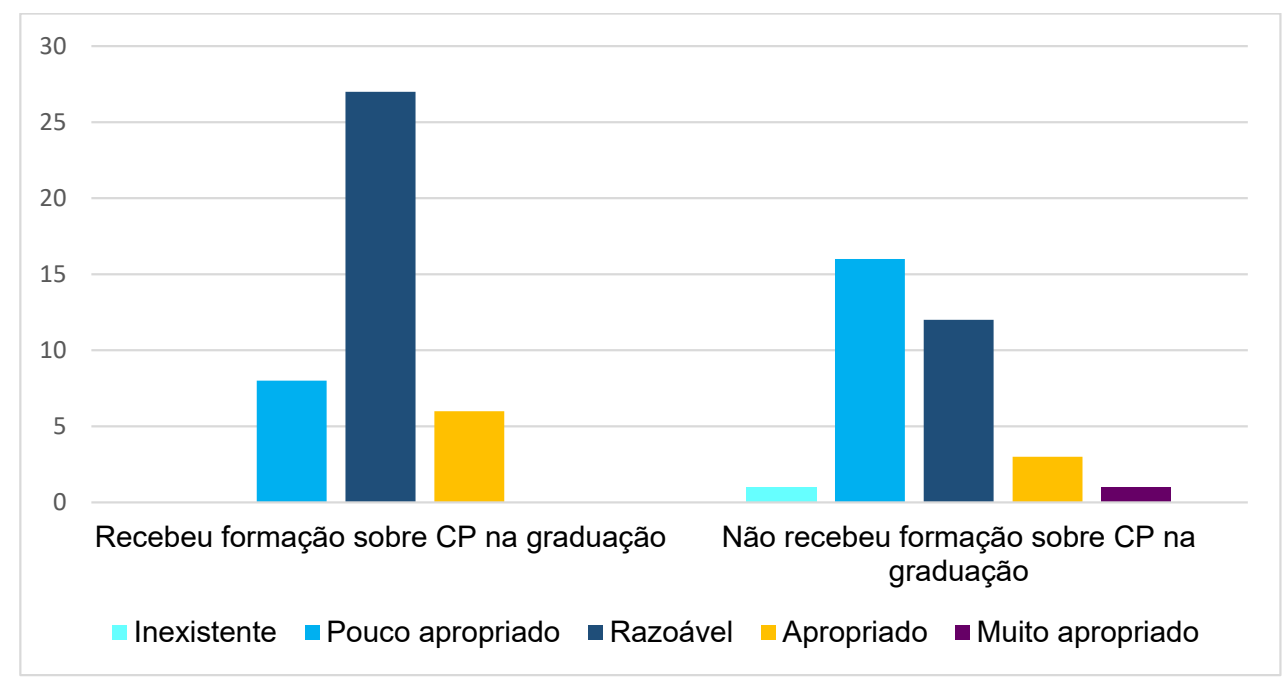


tange àqueles que não receberam formação na graduação $(44,5 \%$, $\mathrm{n}=33)$, a maioria classificou como "pouco apropriado" (48,4\%, $\mathrm{n}=$ 16), enquanto $36,3 \%(n=12)$ consideraram como "razoável".

O Gráfico 2 ilustra a importância da existência do médico em CP, evidenciando que a maioria considera esse tópico"muito importante", ao passo que nenhum participante considerou "nada importante" ou "pouco importante". Entretanto, de acordo com o questionário respondido, apenas 25 participantes $(35,1 \%)$ gostariam de receber formação específica na área, dos quais 18
$(69,2 \%)$ têm interesse em pós-graduação, enquanto sete $(26,9 \%)$ se interessam pela especialidade. Dessa forma, a maioria (64,9\%, $\mathrm{n}=48$ ) informa não ter interesse em atuar nessa área.

A Tabela 3 demonstra a distribuição de erros e acertos para cada uma das 30 perguntas de alternativas de verdadeiro ou falso que identificam os conhecimentos específicos sobre CP. Nota-se que as questões 29 e 30 foram as com maior número de erros, ao passo que as questões 2 , 3, 15, 24, 25 e 26 apresentaram $100 \%$ de acerto.

Gráfico 2.Como o participante enxerga a existência do médico em cuidados paliativos.

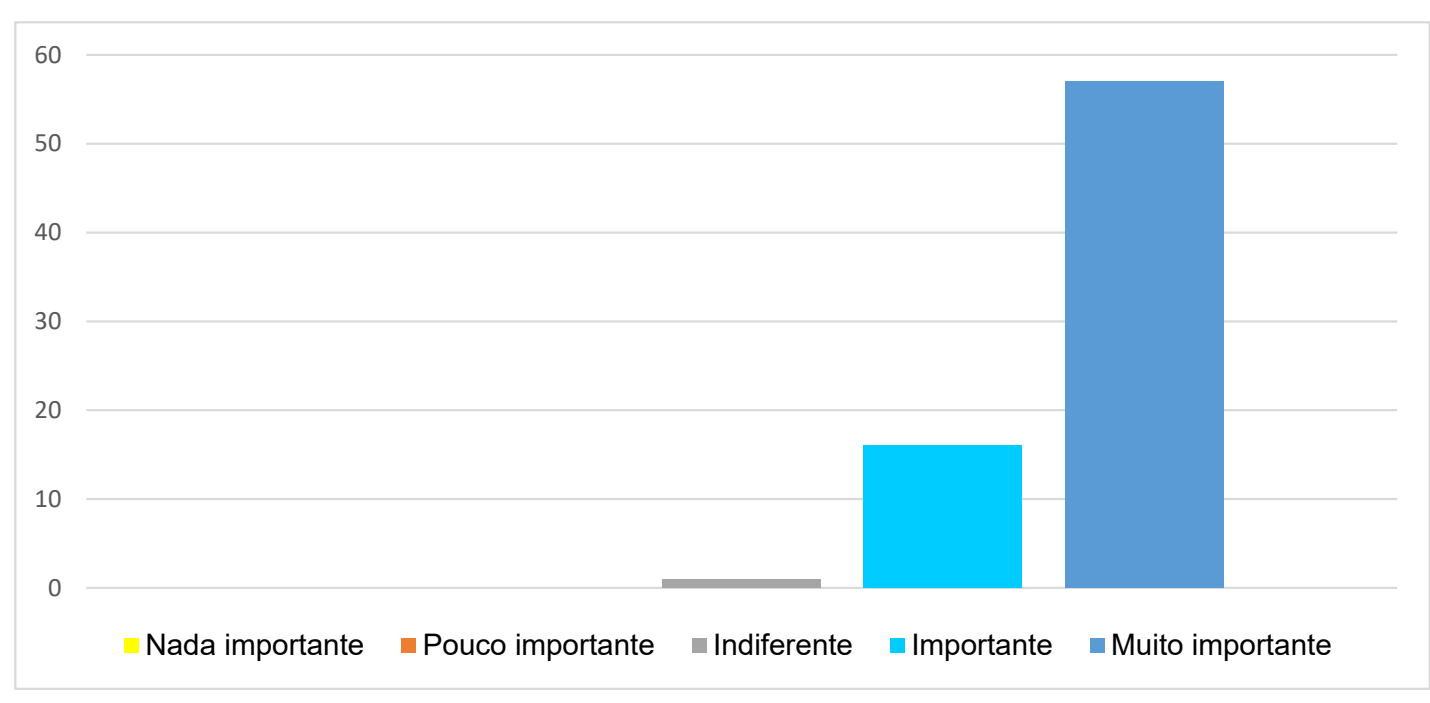

Tabela 3. Erros e acertos evidenciados pelo questionário teórico sobre cuidados paliativos.

\begin{tabular}{|c|c|c|c|c|}
\hline Itens avaliados & Resposta correta & & $\mathbf{n}$ & $\%$ \\
\hline $\begin{array}{l}\text { 1) Os cuidados paliativos são reconhecidos como um elemento essencial } \\
\text { dos cuidados de saúde. }\end{array}$ & V & Acertos & 69 & 93,2 \\
\hline $\begin{array}{l}\text { 2) Os cuidados paliativos constituem uma resposta organizada à } \\
\text { necessidade cuidar dos doentes e apoiá-los na fase final da vida. }\end{array}$ & V & Acertos & 74 & 100 \\
\hline 3) Os cuidados paliativos destinam-se apenas a doentes agônicos. & $\mathrm{F}$ & Erros & 0 & 0 \\
\hline \multirow{2}{*}{ 4) O apoio espiritual não é importante em cuidados paliativos. } & \multirow{2}{*}{$\mathrm{F}$} & Erros & 1 & 1,4 \\
\hline & & Acertos & 73 & 98,6 \\
\hline \multirow{2}{*}{$\begin{array}{l}\text { 5) Os princípios éticos (autonomia, justiça, beneficência e não maleficência) } \\
\text { não se aplicam em cuidados paliativos. }\end{array}$} & \multirow{2}{*}{$\mathrm{F}$} & Erros & 1 & 1,4 \\
\hline & & Acertos & 73 & 98,6 \\
\hline $\begin{array}{l}\text { 7) Quando se determina que a possibilidade de cura não existe, não há mais } \\
\text { nada a fazer pelo doente. }\end{array}$ & $\mathrm{F}$ & Acertos & 72 & 97,3 \\
\hline \multirow{2}{*}{ 8) Os cuidados paliativos preconizam a eutanásia e a distanásia. } & \multirow{2}{*}{$\mathrm{F}$} & Erros & 3 & 4,1 \\
\hline & & Acertos & 71 & 95,9 \\
\hline
\end{tabular}


Tabela 3. (Continuação) Erros e acertos evidenciados pelo questionário teórico sobre cuidados paliativos.

\section{Itens avaliados}

9) Por ação paliativa entende-se qualquer medida com intuito curativo que visa minorar as repercussões negativas da doença sobre o bem-estar global do doente.

10) O principal objetivo dos cuidados paliativos é promover, tanto quanto possível e até ao fim, o bem-estar e a qualidade de vida do doente.

11) Os cuidados paliativos não antecipam nem atrasam a morte.

12) Os cuidados paliativos destinam-se apenas a doentes com idade superior a 65 anos.

13) Os cuidados paliativos podem ser prestados em contexto domiciliário.

14) Doentes com Aids em estádio terminal podem ser admitidos em unidades de cuidados paliativos.

15) Os cuidados paliativos destinam-se apenas a pessoas com doença oncológica.

16) Doente terminal é aquele que apresenta doença avançada, incurável e progressiva e que, em média, tem uma sobrevida esperada de três a seis meses.

17) Ações paliativas e ações curativas devem coexistir intensificando-se as paliativas à medida que a doença vai respondendo menos às curativas.

18) O controle de sintomas não é um dos critérios de admissão em unidades de cuidados paliativos.

19) Náuseas, vômitos e dispneia não são sintomas frequentes em cuidados paliativos.

20) Considera-se como dor apenas a manifestação física dela.

21) Em cuidados paliativos, o tratamento farmacológico da dor não deve seguir a escada analgésica estabelecida pela OMS.

22) Em cuidados paliativos, o objetivo principal é a obtenção da cura, relegando para segundo plano os sintomas predominantes.

23) Na escada analgésica da dor estabelecida pela OMS para a dor moderada (grau 2), são utilizados apenas analgésicos não opioides, como o paracetamol.

24) Dor total compreende aspetos multidimensionais (físicos, psicológicos, sociais e espirituais).

25) Atualmente, não existem instrumentos para avaliação da dor em cuidados paliativos.

26) Para alívio da dor em cuidados paliativos, o médico deve recorrer apenas a medidas farmacológicas

27) Antidepressivos, anticonvulsivantes e neurolépticos são exemplos de coanalgésicos adjuvantes.

28) Os efeitos secundários mais comuns dos opioides são obstipação, náuseas/vômitos, sedação e confusão.

29) A Escala de Edmonton é específica dos cuidados paliativos e avalia os principais sintomas.

30) Em cuidados paliativos, a via de eleição para a administração de terapêutica é a via oral.

\begin{tabular}{|c|c|c|c|}
\hline Resposta correta & & $\mathrm{n}$ & $\%$ \\
\hline \multirow{2}{*}{$\mathrm{F}$} & Erros & 28 & 37,8 \\
\hline & Acertos & 46 & 62,2 \\
\hline \multirow{2}{*}{ V } & Erros & 1 & 1,4 \\
\hline & Acertos & 73 & 98,6 \\
\hline \multirow{2}{*}{ V } & Erros & 2 & 2,7 \\
\hline & Acertos & 72 & 97,3 \\
\hline \multirow{2}{*}{$F$} & Erros & 1 & 1,4 \\
\hline & Acertos & 73 & 98,6 \\
\hline \multirow{2}{*}{ V } & Erros & 1 & 1,4 \\
\hline & Acertos & 73 & 98,6 \\
\hline \multirow{2}{*}{ V } & Erros & 3 & 4,1 \\
\hline & Acertos & 71 & 95,9 \\
\hline \multirow{2}{*}{$F$} & Erros & 0 & 0 \\
\hline & Acertos & 74 & 100 \\
\hline \multirow{2}{*}{ V } & Erros & 23 & 31,1 \\
\hline & Acertos & 51 & 68,9 \\
\hline \multirow{2}{*}{ V } & Erros & 4 & 5,4 \\
\hline & Acertos & 70 & 94,6 \\
\hline \multirow{2}{*}{$F$} & Erros & 5 & 6,8 \\
\hline & Acertos & 69 & 93,2 \\
\hline \multirow{2}{*}{$\mathrm{F}$} & Erros & 7 & 9,5 \\
\hline & Acertos & 67 & 90,5 \\
\hline \multirow{2}{*}{$F$} & Erros & 1 & 1,4 \\
\hline & Acertos & 73 & 98,6 \\
\hline \multirow{2}{*}{$F$} & Erros & 9 & 12,2 \\
\hline & Acertos & 65 & 87,8 \\
\hline \multirow{2}{*}{$F$} & Erros & 2 & 2,7 \\
\hline & Acertos & 72 & 97,3 \\
\hline \multirow{2}{*}{$F$} & Erros & 13 & 17,6 \\
\hline & Acertos & 61 & 82,4 \\
\hline \multirow{2}{*}{ V } & Erros & 0 & 0 \\
\hline & Acertos & 74 & 100 \\
\hline \multirow{2}{*}{$F$} & Erros & 0 & 0 \\
\hline & Acertos & 74 & 100 \\
\hline \multirow{2}{*}{$F$} & Erros & 0 & 0 \\
\hline & Acertos & 74 & 100 \\
\hline \multirow{2}{*}{ V } & Erros & 2 & 2,7 \\
\hline & Acertos & 72 & 97,3 \\
\hline \multirow{2}{*}{ V } & Erros & 2 & 2,7 \\
\hline & Acertos & 72 & 97,3 \\
\hline \multirow{2}{*}{$\mathrm{F}$} & Erros & 50 & 67,6 \\
\hline & Acertos & 24 & 32,4 \\
\hline \multirow{2}{*}{ V } & Erros & 30 & 40,5 \\
\hline & Acertos & 44 & 59,5 \\
\hline
\end{tabular}




\section{DISCUSSÃO}

O presente estudo avaliou o conhecimento teórico dos recém-formados do curso de Medicina da instituição pesquisada sobre CP. Os resultados evidenciaram que a maior parte dos recém-graduados tem formação em $\mathrm{CP}$ durante a graduação em Medicina, porém eles não consideram seu conhecimento apropriado para o exercício médico na área. Além disso, os recém-formados acreditam que a informação oferecida sobre o tema não foi suficiente e que seja muito importante a inclusão de conteúdo sobre CP no curso de Medicina.

De acordo com Malta et al. ${ }^{5}$, das EMB que disponibilizam algum tipo de ensino em CP, 35,1\% oferecem os CP como disciplina eletiva, e, em apenas $33,9 \%$, o ensino é obrigatório para a conclusão da graduação. Ainda assim, a grande maioria refere-se a temas relacionados à morte, à dor ou à área de oncologia, que, apesar de serem relacionados aos $\mathrm{CP}$, não o abordam em sua totalidade. Para a $\mathrm{OMS}^{8}$, a limitação do ensino e treinamento em $\mathrm{CP}$, como observada nas escolas brasileiras, é o principal empecilho para que o acesso aos $\mathrm{CP}$ seja ampliado. Ademais, vale ressaltar que, conforme descrito na Carta de Praga, os CP são direito dos pacientes, sendo dever do governo estimular a capacitação dos profissionais, para que os pacientes tenham acesso a uma atenção qualificada.

Assim, observa-se que a realidade dos formandos de 2020 em relação ao conhecimento recebido durante a graduação está em consonância com as demais escolas do Brasil, evidenciando que a maioria em algum momento teve contato com conteúdo sobre CP. Em relação à instituição de ensino superior analisada, os estudantes têm contato com o tema de maneira pontual em alguns módulos. Porém, assim como avaliado no estudo de Oliveira et al. ${ }^{9}$, esse conhecimento oferecido é pouco sistematizado e não ocorre como uma disciplina específica ou por meio de um ensino longitudinal, como forma de ensino de longo prazo.

Observou-se ainda que $86,5 \%(n=64)$ dos avaliados não consideraram seu conhecimento acerca de CP como "apropriado" ou "muito apropriado". Assim, esses dados corroboram o estudo de Alves Jr. et al. ${ }^{10}$ que abordou estudantes de Medicina em relação à capacidade de prestar $\mathrm{CP}$, e somente a minoria (42,7\%) afirmou estar apta a oferecê-los. Dessa forma, a sensação do médico de ser apto a atender um paciente que necessita de CP é algo de extrema importância social e de saúde pública, pois são pacientes fora de possibilidades terapêuticas, que já se sentem despersonalizados e sofrem ainda mais com a falta de preparo no sistema de saúde.

De acordo com Caldas et al. ${ }^{11}$, conteúdos sobre CP devem ser implementados na base curricular como uma matéria única ou inseridos em diversos componentes do curso. Sendo assim, pode ser feito em um curto espaço de tempo ou por meio de um ensino longitudinal ${ }^{9}$. O estudo de Aranha ${ }^{12}$ defende a criação de uma disciplina específica sobre $C P$, já que é um tema muito atual e com aplicações em todas as áreas da medicina. Além disso, essa incorporação permite aprimorar qualidades nos futuros médicos como humanização e comunicação, áreas que são, infelizmente, pouco exploradas durante a graduação.

$\mathrm{Na}$ atual pesquisa, percebe-se que, mesmo tendo contato com CP durante a graduação médica, a grande parte dos recém-formados $(71,6 \%, \mathrm{n}=53)$ considera muito importante a inclusão de conteúdos sobre $\mathrm{CP}$ no bacharelado de Medicina. Entretanto, a maioria $(64,9 \%, \mathrm{n}=48)$ afirma não ter interesse em atuar nessa área. De acordo com Orth et al. ${ }^{13}$, essa relação ocorre porque, apesar de os participantes reconhecerem a importância do tema em suas carreiras, muitos não têm interesse na área, provavelmente porque percebem o quão complexo é dar assistência necessária a um paciente que necessita de $\mathrm{CP}$ e à sua família.

Quanto às respostas referentes ao questionário de verdadeiro ou falso aplicado na pesquisa, de forma geral a maioria das questões teve mais acertos do que erros, o que indica que o contato com o tema durante a graduação deu aos médicos recém-formados uma boa noção sobre algumas práticas em CP. Ademais, apesar de duas questões apresentarem grande número de erros, apenas uma questão teve mais respostas erradas do que certas.

A questão 29 - "A escala de Edmonton é específica dos cuidados paliativos eavalia os principais sintomas"-éum exemplo de equívoco dos participantes. Isso ocorre porque, de acordo com Nascimento ${ }^{14}$, essa escala é um instrumento de avaliação de sintomas psicológicos e físicos associados aos pacientes oncológicos. Por fim, a questão 30 - "Em cuidados paliativos, a via de eleição para a administração de terapêutica é a via oral" - também apresentou grande número de erros $(40,5 \%, n=3)$. Segundo as diretrizes da OMS para os manejos farmacológico e radioterápico da dor oncológica em adultos e adolescentes ${ }^{15}$, a administração oral é preferível sempre que possível, a fim de evitar custos elevados, intercorrências e desconfortos

Esses resultados demonstram que os temas discutidos durante a faculdade estão pouco voltados para aspectos práticos como anamnese e terapêutica. Outros estudos também demonstram resultado semelhante, como o de Malta et al. ${ }^{5}$, em que se observaram que os médicos consideraram a formação em CP recebida durante o curso de Medicina como insuficiente e muito focada em temas bioéticos, deixando de lado outros aspectos importantes, como a multidisciplinaridade e a farmacologia.

Dalpai et al. ${ }^{16}$ chegaram à conclusão parecida em estudo no qual $89,4 \%$ dos entrevistados afirmaram ter recebido informações insuficientes sobre o atendimento de pacientes 
em situação terminal. Quanto aos sintomas mais comuns, como dispneia, vômito, caquexia e constipação, 80,9\% mencionaram que não receberam explicações suficientes sobre eles e que, por conta disso, não tinham conhecimento teórico. Assim, percebe-se que os dados encontrados no presente estudo corroboram os achados da literatura, uma vez que a minoria dos participantes $(13,5 \%, n=10)$ respondeu que as informações sobre o cuidado com doentes terminais recebidas durante a graduação foram suficientes para a atuação médica.

Esses resultados demonstram a necessidade de uma melhor sistematização do currículo médico no que se refere ao tema dos $C P$, entendendo que um profissional recémformado deve estar apto a prestar cuidados ao paciente nos diversos aspectos, como controle da dor, promoção de conforto e independência, manutenção de atividades importantes para o cotidiano do paciente, além de saber refletir sobre a terminalidade da vida, algo comum durante o acompanhamento. Além disso, a atitude médica positiva ou negativa reflete diretamente no doente e em sua família ${ }^{10}$.

Esta pesquisa teve como limitação a adesão dos participantes por conta da dificuldade imposta pela coleta eletrônica. Apesar disso, acredita-se que este trabalho possa contribuir para significativas reflexões acerca do ensino em CP e melhorias na formação médica, como a ampliação de propostas para a implementação da disciplina específica de $C P$, em curto prazo, ou de forma sistematizada e longitudinal.

Logo, este estudo tem sua importância, pois, muito além de abordar os temas de $\mathrm{CP}$, poderá ter uma contribuição na construção de novos conhecimentos e um possível avanço na qualidade da graduação médica.

\section{CONCLUSÕES}

Diante dos dados obtidos referentes ao questionário aplicado, ratifica-se que o espaço curricular para a abordagem dos CP permanece com grandes carências. Os resultados revelam que, embora os acadêmicos reconheçam a importância dos CP, a falta de informação acerca da sua filosofia e de seus princípios e a insegurança quanto às condutas a serem prescritas destacam a demanda de uma educação continuada sobre o assunto.

Ademais, esses achados corroboram estudos nacionais e internacionais que afirmam a necessidade da disseminação do conhecimento sobre a ideologia dos $C P$, de modo que é um processo que precisa perpassar pela formação médica, a fim de se estender à sua prática. Acrescentar o ensino de CP permanente é um pressuposto importante para efetivar boas práticas, de modo a favorecer atitudes e decisões assertivas e humanizadas.
Em síntese, a partir do presente estudo, vislumbrase na educação a possibilidade de formar médicos não apenas especialistas em $\mathrm{CP}$, mas também profissionais aptos para prestar assistência a pacientes que necessitam de $C P_{1}$ oferecendo conforto e melhoria da qualidade de vida num cenário crescente de doenças crônicas no Brasil.

\section{CONTRIBUIÇÃO DOS AUTORES}

Natália Sousa Costa, Nathália Maria Fonseca, Isabella Augusto Santos, Gustavo Marques Paulino e Júlia Oliveira Carvalho participaram do planejamento da pesquisa, da coleta de dados e da redação do artigo. Aila Davis Fanstone Pina Vieira foi responsável pelo planejamento e pela supervisão da pesquisa, e pela revisão do texto.

\section{CONFLITO DE INTERESSES}

Declaramos não haver conflito de interesses.

\section{FINANCIAMENTO}

Declaramos não haver financiamento.

\section{REFERÊNCIAS}

1. World Health Organization. Definition of palliative care. Geneva:WHO; 2002.

2. Lemos CFP, Barros GS, Melo NCV, Amorim FF, Santana ANC. Avaliação do conhecimento em cuidados paliativos em estudantes durante o curso de Medicina. Rev Bras Educ Med. 2017;41(2):278-82.

3. Academia Nacional de Cuidados Paliativos. Critérios de qualidade para os cuidados paliativos no Brasil. São Paulo: ANCP; 2012 [acesso em 2 set 2019]. Disponível em: https://paliativo.org.br/download/manual-decuidados-paliativos-ancp/.

4. Costa AP, Poles K, Silva AE. Formação em cuidados paliativos: experiência de alunos de medicina e enfermagem. Interface (Botucatu). 2016;20(59):1041-52.

5. Malta R, Rodrigues B, Priolli DG. Paradigma na formação médica: atitudes e conhecimentos de acadêmicos sobre morte e cuidados paliativos. Rev Bras Educ Med. 2018;42(2):34-44.

6. Braun UK, Gill AC, Teal CR, Morrinson LJ. The utility of reflective writing after a palliative care experience: can we assess medical students' professionalism? J Palliat Med. 2013;16(11):1342-9.

7. Lopes SAP. Cuidados paliativos: conhecimentos dos estudantes de licenciatura em Enfermagem [dissertação]. Viseu, Portugal: Escola Superior de Saúde de Viseu; 2013.

8. World Health Organization. Palliative care fact sheet. Geneva: WHO; 2015.

9. Oliveira JR, Ferreira AC, Rezende NA, Castro LP. Reflexões sobre o ensino de bioética e cuidados paliativos nas escolas médicas do estado de Minas Gerais. Rev Bras Educ Med. 2016;40(3):364-73.

10. Alves Jr. VD, Fonseca SR, Gutterres DB, Souza MCA de. Cuidados paliativos: conhecimento de estudantes de graduação em Enfermagem e em Medicina. Revista de Saúde. 2019;10(2):7-11.

11. Caldas GHO, Moreira SNT, Vilar MJ. Cuidados paliativos: uma proposta para o ensino da graduação em Medicina. Rev Bras Geriatr Gerontol. 2018;21(3):261-71.

12. Aranha $A B$. Implementação da disciplina de cuidados paliativos na graduação em Medicina da Universidade Federal do Rio Grande do Norte (UFRN). 2019. 50f. Dissertação (Mestrado A do conhecimento de estudantes de medicina sobre morte encefálica). Rev. Bras. Ter. Intensiva. 2019; 19(2):144-50. 
13. Orth LC, Haragushiku EY, Freitas ICS, Hintz MC, Marcon CEM, Teixeira JF. Conhecimento do acadêmico de Medicina sobre cuidados paliativos. Rev Bras Educ Med. 2020;43 (1 sup 1):286-95.

14. Nascimento JCC. Avaliação da dor em paciente com câncer em cuidados paliativos à luz da literatura. Saúde \& Ciência em Ação. 2017;3(1):11-26.
15. World Health Organization. WHO Guidelines for the pharmacological and radiotherapeutic management of cancer pain in adults and adolescents. Geneva; WHO; 2018.

16. Dalpai D, Mendes FF, Asmar JAVN, Carvalho PL, Loro FL, Branco A. Pain and palliative care: the knowledge of medical students and the graduation gaps. Rev Dor. 2017;18(4):307-10. 